\title{
LÓGICA TRASCENDENTAL Y LÓGICA NATURALISTA
}

\section{Trascendental Logic and Naturalistic Logic}

\author{
Javier CAStro Albano ${ }^{a, b}$ \\ https://orcid.org/0000-0001-8504-929X \\ javiercastroalbano@gmail.com \\ ${ }^{a}$ Universidad de Buenos Aires, Buenos Aires, Argentina \\ ${ }^{b}$ Universidad Nacional de Rosario, Rosario, Argentina
}

\section{Resumen}

Se exponen algunas de las reflexiones de Alberto Moretti acerca del carácter trascendental de la lógica, poniendo especial atención en cierta evolución que parece haber en sus ideas sobre este asunto entre la publicación en el año 2006 de "Lógica y semántica" y "la Lógica y la trama de las cosas", del año 2016. Se presentan los rasgos más generales de una versión del naturalismo lógico que podría sobrevivir a los cuestionamientos trascendentalistas de Moretti.

Palabras clave: Lógica trascendental; Lógica naturalista; Principios lógicos.

\begin{abstract}
Some of Alberto Moretti's thoughts about the trascendental nature of logic are presented, with special emphasis in the evolution of his ideas about this subject between the publication in 2006 of "Lógica y semántica" and "La lógica y la trama de las cosas" from 2016. The most general traits of a version of logical naturalism that might survive Moretti's transcendentalist objections is presented.
\end{abstract}

Key words: Transcendentalism about Logic; Naturalism about Logic; Logical Principles.

"Llamemos naturalista", sugiere Alberto Moretti, "al punto de vista según el cual toda teoría, en última instancia, es teoría empírica, y no hay nada que sea mostrable por el uso del lenguaje y no pueda ser dicho por una tesis empíricamente evaluable" (2006). Esta caracterización decididamente me convierte en un naturalista. Lo que no sería un problema, si no fuera porque Moretti también ha argumentado que no todo lo que es "mostrable por el uso del lenguaje" puede ser atrapado en las redes del naturalista. En particular, Moretti entiende que hay un aspecto de la lógica de un lenguaje natural que siempre va a eludir al naturalista, pero al que pueden llegar a acceder 
aquellos que son capaces de contemplarla desde un punto de vista trascendental.

La mayor parte de este trabajo está dedicada a exponer las ideas de Moretti acerca del carácter trascendental de la lógica, poniendo especial atención en cierta evolución que parece haber en sus ideas sobre este asunto entre la publicación en el año 2006 de "Lógica y semántica" y "la Lógica y la trama de las cosas", del año 2016. En la sección final intentaré delinear los rasgos más generales de una versión del naturalismo lógico que quizás pueda sobrevivir a los cuestionamientos trascendentalistas de Moretti.

\section{Lenguaje y lógica}

"El habla, la capacidad de intercambio lingüistico, es una precondición de nuestro modo de ser", sostiene Moretti, "No sorprende entonces que la noción de lenguaje aparezca de manera normal, al hablante típico, para circunscribir los que parecen fenómenos lingüisticos paradigmáticos, en un intento por tomar conciencia de este modo de ser en el habla" (2006). Moretti señala aquí el que suele ser el principal objetivo del estudio filosófico de los "fenómenos lingüisticos": iluminar de algún modo "este modo de ser en el habla" característico de "la etapa más reciente de la experiencia humana". Y también deja claro que ese estudio de los "fenómenos lingüísticos" depende crucialmente de "la noción de lenguaje". Pero el hecho de que la noción de lenguaje "aparezca de manera normal, al hablante típico" no debería hacernos olvidar que se trata de una noción teórica, una construcción con la que intentamos capturar nuestro genuino objeto de estudio: "el habla, la capacidad de intercambio lingüístico". El lenguaje no se da a nuestra experiencia. Es la conducta humana la que tenemos ante nosotros y la noción teórica de lenguaje es una herramienta conceptual con cuya ayuda logramos "circunscribir" algunos ejemplos particulares de esa conducta.

Para Moretti, la lógica ocupa un lugar central en nuestros intentos de elucidación de la conducta lingüística. "Cuando se habla del lenguaje como práctica humana en relación con la búsqueda de conocimiento o comprensión se atiende particularmente, y con razón, a la práctica argumentativa" (2006). Pero como hay razones para pensar que atender a este uso del lenguaje orientado a la búsqueda de conocimiento puede ser "el modo apropiado de iniciar la consideración de la práctica lingüística en general, porque cualquier otra intención lingüística se apoya en esta" (2006), la reflexión sobre la práctica argumentativa, la lógica, se convierte en un componente crucial para la reflexión sobre el lenguaje en general. 
La práctica argumentativa consiste en la producción de aserciones. Pero producir aserciones, afirmar, no es meramente emitir oraciones. Quien, al emitir una oración, produce una aserción (afirma algo), se involucra en una práctica que lo compromete (por lo general implícitamente) a seguir una serie de normas constitutivas de esa práctica. Participar de esa práctica supone, por ejemplo, que no podemos afirmar (en el sentido de que no sería correcto hacerlo) ciertas oraciones una vez que hemos afirmado otras, o que estamos obligados (en el mismo sentido) a afirmar ciertas oraciones (o a aceptarlas una vez que otro lo ha hecho) cuando hemos afirmado otras. Estas conexiones entre las oraciones, que resultan de las normas que rigen la producción de aserciones, "exponen los lazos de significación más firmes que hacen posible la interpretación lingüistica intersubjetiva" y se resumen "en algunos principios que sistematizan el uso de términos singulares, predicados y oraciones. Se los llama principios lógicos" (2016). Los principios lógicos, pues, exponen las conexiones entre las oraciones que reflejan en el lenguaje las normas que constituyen la práctica de producir aserciones. En consecuencia: "Desarrollar la reflexión lógica es explicitar (hipotéticamente) las determinaciones de la posibilidad de aseverar, las condiciones que hacen posible la aserción" (2006).

Hay dos rasgos que Moretti le asigna a esta investigación sobre "las condiciones que hacen posible la aserción" que, para nuestros presentes fines, es importante remarcar. Uno de ellos tiene que ver con el origen de esta investigación y el otro con su naturaleza. En cuanto al origen, Moretti entiende que la reflexión lógica "es resultado del esfuerzo natural por mejorar nuestra comprensión de [la práctica de evaluar razonamientos], con la esperanza de mejorar también su ejercicio. El esfuerzo responde tanto a motivos "prácticos" (hay casos de discrepancia y casos de incertidumbre evaluativa) cuanto a motivos "teóricos" (descubrir, explicitar, los criterios regulativos de la evaluación)" (2006). En cuanto a su naturaleza, Moretti ha insistido a lo largo de su obra sobre la existencia de un "nexo fundamental entre reflexión lógica y reflexión semántica” (2006).

Para lo que sigue puede ser útil esquematizar un poco la imagen de la investigación lógica que se desprende de los párrafos anteriores. Supongamos que un grupo de personas preocupadas por "la búsqueda de conocimiento o comprensión" de algún aspecto del mundo ha llegado a formular la teoría T (que no debería considerarse, en este punto, como algo más que un conjunto de oraciones del lenguaje que el grupo de investigadores ha estado usando "con pretensiones cognoscitivas"). Supongamos, también, que no existe entre las personas involucradas en 
la investigación ni incertidumbre ni discrepancia sobre el hecho de que algunas oraciones de $\mathrm{T}$, tomadas conjuntamente, implican lógicamente a la oración $\mathrm{A}$, pero que sí existe discrepancia o incertidumbre sobre si otra oración, B, está implicada lógicamente por (algunas oraciones de) la teoría T. De manera que los investigadores coinciden en que la teoría $\mathrm{T}_{\mathrm{A}}$, que resulta al extender $\mathrm{T}$ sumándole $\mathrm{A}$, mejora, de algún modo, su "conocimiento o comprensión" del mundo, pero no terminan de decidirse sobre si es igualmente conveniente extender T, o $\mathrm{T}_{\mathrm{A}}$, sumando B. En un contexto como este, la reflexión lógica podría surgir impulsada por el deseo de resolver la discrepancia o la incertidumbre de los investigadores respecto de $\mathrm{B}$ (la motivación práctica) o por el deseo de comprender mejor el criterio que ha estado implícitamente regulando la investigación y que, por ejemplo, ha legitimado la adición de A a T (la motivación teórica). Las dos cuestiones están conectadas: la plausibilidad de una demarcación rigurosa entre la validez y la invalidez lógicas propuesta para dar cuenta de los casos problemáticos como el generado por B dependerá, en parte, de lo bien que recoja las opiniones de los participantes de la práctica respecto de los casos no problemáticos como el de $\mathrm{A}$. Esa reflexión lógica comenzará identificando el lenguaje $\mathrm{L}_{\mathrm{T}}$, el lenguaje subyacente a la teoría $\mathrm{T}$ (que debería incluir todas las oraciones que son parte de la teoría $\mathrm{T}$ y otras oraciones que, como A y B, son relevantes para la investigación que estaba en marcha) y se esforzará por establecer una serie de resultados semánticos que iluminen "las condiciones que hacen posible la aserción" de oraciones $\mathrm{L}_{\mathrm{T}}$.

Una cuestión que ha preocupado a Moretti a lo largo de su obra sobre lógica es: ¿qué tipo de reflexión puede ofrecer el tipo de aclaración filosófica que buscamos de "las condiciones que hacen posible la aserción" de oraciones $\mathrm{L}_{\mathrm{T}}$ ?

\section{Lógica sin teorías lógicas}

"Teorizar", observa Moretti en "Lógica y semántica", es "nuestro modo característico de comprender", por lo que es natural esperar que "surja la intención de construir una teoría alrededor de ese concepto [el de lenguaje]" (2006). Llamemos $\mathrm{S}_{\mathrm{LT}}$ a una teoría semántica con la que se pretende dar cuenta de la lógica del lenguaje $\mathrm{L}_{\mathrm{T}}$, el lenguaje subyacente a la teoría T. En sus versiones habituales $\mathrm{S}_{\mathrm{LT}}$ se formula en el metalenguaje de $\mathrm{L}_{\mathrm{T}}$. ¿Puede una teoría como $\mathrm{S}_{\mathrm{LT}}$ alcanzar los objetivos que Moretti le ha asignado a la reflexión lógica? Moretti responde: "no puede haber propiamente teoría lógico-semántica. Teoría en el sentido de fundamentación o explicación en términos más básicos” (2006). 
Esta imposibilidad parece seguirse de consideraciones como las siguientes. Según lo que se ha visto, para Moretti el objetivo de la reflexión lógica es iluminar "las condiciones que hacen posible la aserción" de oraciones $\mathrm{L}_{\mathrm{T}}$. Pero "las condiciones que hacen posible la aserción" son también las que hacen posible la formulación de teorías. La práctica argumentativa normalmente no se manifiesta en la producción de aserciones aisladas, sino en la producción de teorías. Que no son otra cosa que sistemas de oraciones vinculadas por conexiones lógicas. Pero entonces es imposible que una teoría pueda explicar o justificar en términos más básicos las condiciones que hacen posible el discurso teórico. La propia teoría $\mathrm{S}_{\mathrm{LT}}$ requiere para su formulación, para la demostración de sus teoremas, una lógica de la que ella misma no puede dar cuenta: " $L a$ reflexión lógica opera conforme a principios, y cuando se produce en un metalenguaje distinto del lenguaje examinado, algunos de esos principios se verán reflejados en los axiomas y reglas que se especifiquen, pero no todos ellos. Por ejemplo, aserciones como "Si A, entonces A" y reglas como el modus ponens se encontrarán tanto en el metalenguaje como en su lenguaje-objeto, pero aserciones como "El modus ponens es una regla lógica del lenguaje L", o "'Si A, entonces A' y 'Si es necesario que A, entonces A' son leyes lógicas de L", son propias del metalenguaje e innecesarias en el lenguaje-objeto. Esta circunstancia hace extraña la empresa reflexiva, esto es, "auto-referente", de especificar los principios con que opera la propia indagación lógica”. (2006).

¿En qué consiste, pues, la reflexión lógica, si no se trata de una reflexión teórica orientada a producir explicaciones o justificaciones? En "Lógica y semántica" Moretti conecta de una manera original ideas de Frege y de Davidson para ofrecer una respuesta novedosa para esta antigua pregunta. El punto de partida es la tesis de que "El objetivo de este decir [el de la reflexión lógica] no es aumentar nuestro conocimiento teórico sino afianzar una práctica” (2006). La reflexión lógica, en opinión de Moretti, no debe entenderse "como genuina teoría sino como recurso para adquirir una habilidad. Dicho de otro modo: aparece como la constitución de un marco dentro del cual formular teorías, esto es, como dando la posibilidad de teorizar y, por ende, no como teoría" (2006). ¿Cómo consigue la lógica este resultado? ¿Cómo contribuye la lógica a afianzar la práctica de formular teorías? La reflexión lógica consigue este objetivo produciendo textos que, sin ser teorías, parecen serlo: "lo que tenemos no es una teoría sino sólo un modo aparentemente teórico de mostrar el funcionamiento correcto del lenguaje" (2006). Este poder iluminador de algo que tiene la apariencia de teoría, sin serlo, se explica porque, como ya se ha mencionado, teorizar es "nuestro modo 
característico de comprender". "Que una apariencia de teoría sea útil", confirma Moretti, "se basa en que la teorización es nuestra manera típica de aprender" (2006).

El resultado de la reflexión lógica, según este modo de entenderla, "aunque no tiene carácter informativo no es inútil porque puede ayudar a ver, puede orientar la mirada hacia ese carácter constitutivo" (2006) que poseen los principios lógicos. La capacidad de "orientar la mirada" que posee el producto de la reflexión lógico-semántica no se deriva de que en él se describa o asevere o informe sobre los principios lógicos que usan los hablantes de $\mathrm{L}_{\mathrm{T}}$. "Admitido que describir, aseverar, informar, no es todo lo que nuestro lenguaje común permite. Wittgenstein (cualquiera de ellos) retomó con su habitual intensidad uno de los núcleos, más o menos implícito, de las reflexiones lógico-semánticas de Frege, cuando sostuvo que la lógica (la semántica, la gramática) es trascendental” (2006).

\section{Pensar en algo sin entenderlo como algo}

En "La lógica y la trama de las cosas" Moretti ofrece una visión de la dimensión trascendental de la reflexión lógica que va más allá de la presentada en "Lógica en semántica". Todavía considera, como en "Lógica y semántica", que la lógica tiene un carácter constitutivo del uso del lenguaje con fines cognoscitivos: "el lenguaje (el ser hablante) no es posible sin conexiones necesarias entre oraciones (sin normas básicas para la emisión de oraciones)" (2016), conexiones que se resumen en los principios lógicos. Pero ahora también observa que, al constituir el lenguaje, la lógica también constituye al mundo: "los principios lógicos también exponen y determinan una estructura elemental para lo que llamamos mundo o realidad" (2016). De manera que "tanto el discurso cognoscitivo como aquello cognoscible discursivamente, están constituidos por principios lógicos” (2016).

Además de esta doble función de los principios lógicos, Moretti introduce otras dos novedades de importancia en "La lógica y la trama de las cosas": la primera tiene que ver con la explicita oposición al punto de vista naturalista de la lógica; la segunda, con la reconsideración del rol que tiene una teoría semántica como $\mathrm{S}_{\mathrm{LT}}$ en la caracterización de los principios lógicos que constituyen simultáneamente al lenguaje $\mathrm{L}_{\mathrm{T}} \mathrm{y}$ al (fragmento del) mundo del que habla $\mathrm{L}_{\mathrm{T}}$. En "Lógica y semántica" Moretti no objetaba específicamente al naturalismo sino, de manera más general, a cualquier proyecto que ofreciera teorías que pretendieran explicar o justificar los principios lógicos. En "La lógica y la trama de las cosas" la situación es diferente: "Esa constitución de mundo" sostie- 
ne ahora Moretti, "tiene dos aspectos. Por una parte, la constitución de la posibilidad de un mundo y, por ende, un aspecto "independiente" del mundo efectivo y del lenguaje o sublenguaje específico. Procuremos expresarlo mediante la "tesis": hay principios lógicos. [...] Por otra parte, la constitución de un mundo efectivo, que no deriva del aspecto anterior [...] y que se explicita, en su nivel más general y estable, con la construcción, empíricamente regulada [...] de múltiples teorías lógico-semánticas que, a la vez, explicitan y estatuyen específicos principios lógicos” (2016). Una construcción como $\mathrm{S}_{\mathrm{LT}}$, entendida ahora como genuina teoría semántica naturalista, tiene una función que cumplir en la lógica: ella participa de la constitución del segundo aspecto de lenguaje y mundo, el momento en que se "explicitan y estatuyen" los principios lógicos que constituyen a la vez el "mundo efectivo" y las condiciones de aserción del lenguaje específico con el que teorizamos sobre él.

¿A qué se debe esta concesión al naturalista que estaba ausente de "Lógica y semántica"? Quizás la clave para responder a esta pregunta se encuentre en un pasaje de la "La lógica y la trama de las cosas" en donde Moretti reconoce que, para un naturalista, la justificación de una aserción "reside en su papel dentro de teorías empíricamente revisables" (2016). Para el naturalista, esto vale para todas las aserciones teóricas y, en particular, para los principios lógicos. De manera que el naturalista dispone de una estrategia para justificar los principios lógicos: mostrar su relevancia para la derivación de los enunciados de observación que justifican nuestras mejores teorías.

Sin embargo, este rol que Moretti le concede a la teoría lógica naturalista en "La lógica y la trama de las cosas", tiene un carácter subalterno. Porque una teoría semántica empírica como $\mathrm{S}_{\mathrm{LT}}$ no está en condiciones de capturar el aspecto más fundamental del rol constitutivo de la lógica, el aspecto en el que se pretende dar cuenta de las condiciones de posibilidad de la posesión de un lenguaje y de un mundo "independiente' del mundo efectivo y del lenguaje o sublenguaje específico".

¿Por qué no puede el naturalista aspirar a producir una teoría sobre este aspecto fundamental de la constitución de lenguaje y mundo? Moretti responde: "las reflexiones naturalistas sobre las presuposiciones del hablar significativo acerca de la realidad resultan incompletables" (2016). Es esta imposibilidad que tiene el naturalista de completar su teoría sobre las condiciones de posibilidad del uso asertivo del lenguaje lo que impide que pueda dar cuenta del carácter constitutivo de la lógica. Esta imposibilidad tiene dos causas. Una de ellas tiene que ver con el hecho de que para el naturalista la semántica, como cualquier otra teoría, está compuesta de aserciones, por lo que una teoría semántica que 
pretenda iluminar las condiciones de posibilidad de la aserción debería dar cuenta también de sus propias aserciones. Sin embargo, "los esfuerzos de hecho realizados para construir una teoría lógico-semántica general, aplicable también al lenguaje de la teoría misma, se han mostrado hasta ahora ineficaces. Han necesitado o empleado conceptos y afirmaciones metalingüisticas que no pueden reflejarse en el lenguaje-objeto. Con el metalenguaje se ven propiedades del lenguaje-objeto que no pueden verse en el metalenguaje si no es con ayuda de un meta-metalenguaje" (2016). Este punto ya había sido mencionado en "Lógica y semántica" y estaba en el origen de la propuesta de considerar a los productos de la reflexión lógica no como teorías compuestas de aserciones sino como construcciones que resultan de un uso no asertivo del lenguaje. La otra causa de incompletitud no había sido señalada en "Lógica y semántica" y tiene que ver con la combinación de la idea, propia del naturalismo, de que la aceptabilidad de los principios lógicos "es siempre empíricamente disputable y evaluable en términos de un necesariamente abierto criterio de ajuste a la experiencia intersubjetiva" con el hecho de que "no hay manera de anticipar la forma en que se proyectarán las prácticas intersubjetivas (en particular, la práctica de elaborar creencias sobre el mundo)" (2016). Las dos causas mencionadas son dos aspectos del mismo problema: la dificultad del naturalismo para identificar principios que puedan ser considerados constitutivos del discurso asertivo en general. En el primer caso, porque los principios de los que dispone no alcanzan a las aserciones con las que se formula la teoría semántica; en el segundo caso, porque las aserciones que están en condiciones de ser revisadas en el contexto de la práctica argumental no pueden aspirar a ser principios constitutivos de esa práctica.

Lo más cerca que podemos llegar de expresar nuestra captación de este aspecto más básico de la constitución del mundo y el lenguaje, o de sugerir lo que hemos llegado a captar, es por medio de oraciones como "Hay principios lógicos", que pretenden señalar la centralidad de la lógica en la constitución simultanea de mundo y lenguaje. Pero esta es una manera muy defectuosa de hacerlo: "los principios lógicos específicos implícitamente establecen, entre otros significados, el significado general de la palabra 'hay', y las condiciones generales de toda teoría formulable en ese lenguaje. Entonces, cuando pretendemos expresar las condiciones que hacen posible que haya discurso y mundo diciendo, como hace un momento, "hay principios lógicos" enfrentamos una dificultad. Si la "tesis" de que hay principios lógicos [...] es una genuina tesis, su uso de 'hay' está normado por algunos principios lógicos específicos que vertebran el lenguaje en que se formula. Entonces ese 'hay' es el mismo 
que el de la oración 'Hay planicies volcánicas'. Y la tesis queda sujeta a revisión empírica (en tanto su justificación reside en su papel dentro de teorías empíricamente revisables en cualesquiera componentes). Pero entonces no expresa lo que pretendía: condiciones constitutivas y por ende independientes de cualquier lenguaje específico" (2016).

$\mathrm{Al}$ igual que en "Lógica y semántica", en "La lógica y la trama de las cosas" Moretti sostiene que la reflexión lógica fundamental "no califica [...] como genuina teoría" (2016). Pero a diferencia de "Lógica y semántica" en donde, siguiendo la línea de Frege y del Wittgenstein del Tractatus, la opción era aprovechar nuestra familiaridad con el modo de comprender que proporcionan las teorías para construir un texto que, en virtud de su apariencia de teoría, pudiera resultar iluminador, en "La lógica y la trama de las cosas" la situación es otra: la reflexión lógica ahora resulta en "un discurso pretendidamente explicitador de algo que no puede concebirse sino bajo la forma de un hecho efectivo, de una circunstancia del mundo, cuando, al mismo tiempo, se quiere presentar como "algo" [...] que establece la posibilidad de pensar circunstancias mundanas. Se dice que eso es algo conceptualmente anterior a la aparición del concepto de algo. Como decir de lo que se piensa como algo, que no es algo. Y esto, parece claro, no es decir de nada" (2016). El problema ya no es, como en "Lógica y semántica" el de tratar de explicar o justificar la lógica sin presuponer lo mismo que se quiere explicar o justificar. El problema ahora es que la lógica específica, la lógica que norma lo que podemos decir, no nos deja hablar sobre los principios fundamentales que constituyen ese decir. La reflexión lógica ya no se ocupa de orientar la mirada hacia los principios lógicos que tenemos para "afianzar una práctica”, sino que intenta ayudarnos a ver más allá (o más acá) de nuestra práctica. Se trata de "disponer a cierta experiencia peculiar "inefable", que Moretti resume del siguiente modo: "Un acto asertivo es, por lo general, resultado de la experiencia de una circunstancia del mundo. Pero, al mismo tiempo, sugiere el punto de vista trascendentalista, ese acto posibilita una experiencia diferente, la experiencia de esa aserción y de la aserción como tal. Y sobre esa experiencia se puede erigir la idea (sin contenido empírico) del darse del mundo" (2016).

\section{Naturalismo y condiciones de posibilidad de la aserción}

Quienes simpatizan con el naturalismo suelen mirar con suspicacia distinciones como la que traza Moretti entre aquellos principios de una teoría que cuentan como principios constitutivos de la práctica de la aserción y los demás principios. Distinciones como esas, según establece 
la doctrina naturalista "oficial", son vamos intentos por volver a trazar una frontera nítida entre lenguaje y teoría, cuya desaparición habría quedado definitivamente establecida cuando Quine abolió el dogma de la analiticidad. Pero esa no es una respuesta satisfactoria a una posición como la de Moretti, pues esa respuesta depende de que se haya admitido previamente el empirismo (o, al menos, alguna versión de él) y lo que Moretti está impugnando es precisamente el enfoque empirista del lenguaje. Moretti sostiene que la reflexión sobre "los lazos de significación más firmes que hacen posible la interpretación lingüistica intersubjetiva", los llamados principios lógicos, no puede completarse sin "erigir la idea (sin contenido empírico) del darse del mundo". Este es un claro desafío al naturalismo. Insistir en que esa idea no existe por carecer de contenido empírico no constituye una respuesta a Moretti, sino una manera de expresar que no se quiere discutir sobre el asunto.

Una genuina respuesta naturalista a los argumentos de Moretti consistiría, creo que yo, en una defensa de la tesis de que, en contra de lo que Moretti ha sostenido, las herramientas con las que cuenta el naturalismo sí pueden dar cuenta de "los lazos de significación más firmes que hacen posible la interpretación lingüistica intersubjetiva". No es este el lugar para presentar una defensa detallada y completa de esa tesis, pero en los siguientes párrafos voy a exponer la estrategia general que creo que debería seguirse para intentar esa defensa.

Como hemos visto, Moretti sostiene que el naturalismo no puede dar cuenta del rol que los principios lógicos tienen como principios constitutivos de la práctica asertiva porque "las reflexiones naturalistas sobre las presuposiciones del hablar significativo acerca de la realidad resultan incompletables". Moretti ofrece dos argumentos para justificar esa imposibilidad del naturalismo para completar su teoría semántica: uno de ellos, que podemos llamar argumento sincrónico, tiene que ver con la imposibilidad de formular una teoría semántica que pueda dar cuenta de las aserciones de la propia teoría semántica; el otro, que podemos llamar argumento diacrónico, tiene que ver con la imposibilidad de predecir la evolución futura del lenguaje. En líneas muy generales, la estrategia que sugiero para responder al desafío de Moretti consiste en mostrar que, a pesar de que esos argumentos ciertamente afectan a las versiones más típicas del naturalismo lógico, hay una versión del naturalismo lógico que es inmune a ellos.

Comencemos por el argumento sincrónico. Moretti señala que "los esfuerzos de hecho realizados para construir una teoría lógico-semántica general, aplicable también al lenguaje de la teoría misma, se han mostrado hasta ahora ineficaces". Cuando Moretti está hablando 
aquí de "teoría lógico-semántica general", se está refiriendo al tipo de teoría semántica, como la teoría de la verdad de Tarski o la teoría de modelos, cuyos conceptos centrales son los de referencia y verdad. Comparto completamente las dudas de Moretti sobre la posibilidad de producir una teoría lógica basada en conceptos como los de verdad o referencia aplicable al lenguaje de la teoría misma. Pero existe otro enfoque de la teoría semántica, el enfoque inferencialista, que no padece esas mismas limitaciones vinculadas con la autoreferencia. Para el enfoque inferencialista de la lógica, la noción de consecuencia lógica se define en términos sintácticos y, gracias a Gödel, sabemos que un lenguaje puede contener su propia sintaxis. Sugiero, pues, que una aproximación inferencialista a la teoría lógica puede ser útil para bloquear el argumento sincrónico de Moretti. Moretti seguramente no estaría de acuerdo, pues no tiene en muy alta estima al enfoque inferencialista de la lógica, "en vista de los problemas para desechar, sin recurrir a nociones como las de referencia y verdad, casos de definiciones puramente sintácticas del uso de signos que resultan inaceptables como definiciones de signos lógicos" (2016). Moretti se está refiriendo aquí a los conocidos problemas que genera la introducción puramente sintáctica de conectivas como la célebre "tonk", de Arthur Prior. También coincido con Moretti en que los intentos por resolver estos problemas en términos puramente sintácticos no son satisfactorios y que la mejor manera de lidiar con estos casos es aplicando nociones como las de referencia y verdad. ¿No nos lleva esto de regreso al punto de inicio? Yo creo que no, y este es la idea central de la estrategia que propongo para responder al desafío de Moretti. No creo que sea lo mismo utilizar las nociones de referencia y verdad para definir la noción de consecuencia lógica, que utilizarlas "para desechar [...] casos de definiciones puramente sintácticas del uso de signos que resultan inaceptables". El uso de los conceptos de referencia y verdad para definir la noción de consecuencia lógica para un cierto lenguaje L exige que esos conceptos se apliquen a todas las oraciones de L. Es precisamente esta exigencia la que impide que los conceptos de referencia y de verdad de $\mathrm{L}$ sean expresables en L (en virtud de las contradicciones que se producirían). Pero si lo que se quiere es usar los conceptos de referencia y verdad, no para definir la noción de consecuencia lógica para las oraciones de L, sino para legitimar una definición sintáctica de esa noción, entonces quizás la situación sea diferente.

Sea L un lenguaje con la capacidad suficiente como formular los conceptos de referencia y de verdad de un subconjunto propio $\mathrm{R}_{\mathrm{L}}$ de $\mathrm{L}$ (evitando así las contradicciones semánticas conocidas). Es posible formular diferentes tipos de teoría de la verdad "parciales" de acuerdo a 
la extensión que tenga el conjunto $R_{L}$. Mi punto es el siguiente: si $R_{L}$ ha sido elegido de manera apropiada, entonces la prueba de que las reglas de inferencia de $\mathrm{L}$, cuando se restringen a oraciones que pertenecen a $R_{L}$, exhiben el comportamiento apropiado en términos de verdad o referencia (por ejemplo: garantizan la preservación de la verdad), es suficiente para legitimar el uso de esas reglas como reglas lógicas. La cuestión, por supuesto, es entender qué quiere decir la expresión "de manera apropiada" en la oración anterior. Todo el peso de la estrategia que propongo está apoyado en el supuesto de que es posible identificar un subconjunto $R_{L}$ de oraciones de $L$ que sea suficiente para la legitimación de las reglas lógicas que valen para las oraciones del lenguaje entero L. No puedo explicar aquí como creo que debería caracterizarse ese subconjunto $R_{\mathrm{L}}$. Pero confío en que la explicación precedente haya alcanzado para establecer que si fuera posible identificar un subconjunto $R_{L}$ de $L$ que tenga esas características, contaríamos con una manera de producir una teoría lógico-semántica que se aplique al lenguaje de la teoría misma (porque su relación de consecuencia lógica ha sido definida en términos sintácticos), pero que se mantenga apropiadamente conectada con los conceptos de verdad y referencia (para descartar casos inaceptables como el de "Tonk").

El enfoque inferencialista sobre el que se basa la estrategia que he sugerido no es el típico. El inferencialismo típico suele presentarse como una opción frente a una semántica basada en conceptos como los de verdad y referencia y su objetivo teórico suele ser encontrar la manera de producir una teoría semántica satisfactoria en términos puramente sintácticos. Pero el uso de los conceptos de verdad y referencia es crucial en la presente propuesta. Creo que la formulación de una teoría lógica de carácter naturalista requiere una combinación de ambos tipos de enfoque de la semántica.

La justificación de un cierto principio formulable en el lenguaje L (sea un principio lógico o no lógico) resulta, como en cualquier concepción naturalista, de "su papel dentro de teorías empíricamente revisables". Pero lo que hace que ese principio sea un principio lógico del lenguaje $\mathrm{L}$, lo que separa a ese principio de otros (como los inadmisibles principios que se desprenden de la definición de "tonk" o cualquier otro principio más útil pero que no tienen carácter lógico), es su comportamiento en términos de verdad y referencia (que se manifiesta cuando se lo restringe a $R_{L}$ ). Los conceptos de verdad y referencia no intervienen en la justificación de los principios lógicos, sino en la demarcación entre lo lógico y lo no lógico. Los principios llegan a nuestras teorías por su relevancia para la derivación de resultados empíricos. Después de eso 
podemos preguntarnos cuáles de ellos cabe catalogar como principios lógicos y cuáles no. Recién ahí es donde intervienen los conceptos de verdad y referencia, para garantizar que los principios catalogados como lógicos cuentan como determinando "las condiciones que hacen posible la aserción" de oraciones de L.

Ya mencioné que para bloquear el argumento sincrónico es crucial que el fragmento $R_{L}$ de $L$ tenga ciertas características especiales que justifiquen el rol que cumple en la legitimación de la noción de consecuencia lógica, definida sintácticamente, para L entero. Para bloquear el argumento diacrónico se requiere algo más: que la lógica que logra delimitar ese procedimiento sea única. Se requiere una garantía de que cada vez que hay un lenguaje $L$ que cuenta con un fragmento $R_{L}$ que satisface las condiciones para legitimar una noción sintáctica de consecuencia lógica para $L$ y que hay otro lenguaje $L^{*}$ que cuenta con un fragmento $R_{\mathrm{L}^{*}}$ que satisface las condiciones para legitimar una noción sintáctica de consecuencia lógica para $L^{*}$, entonces la noción de consecuencia lógica de $\mathrm{L}$ es la misma que la de $\mathrm{L}^{*}$. Creo que es crucial para responder al argumento diacrónico de Moretti que la lógica sea única y que se mantenga estable a pesar de los cambios que pueda traer la investigación empírica. En esto mi propuesta tampoco es la típica propuesta naturalista, que considera que la aceptabilidad de los principios lógicos es "siempre empíricamente disputable y evaluable en términos de un necesariamente abierto criterio de ajuste a la experiencia intersubjetiva" y que "son posibles sistemas alternativos con el mismo nivel de adecuación [empírica]" (2006). Este naturalismo típico es el que permite la distinción, central para la propuesta trascentalista de Moretti, entre el aspecto de la reflexión lógica que se manifiesta en "la construcción, empíricamente regulada [...] de múltiples teorías lógico-semánticas que, a la vez, explicitan y estatuyen específicos principios lógicos" y el aspecto, más fundamental e inefable, “independiente' del mundo efectivo y del lenguaje o sublenguaje específico". Según creo, la única manera de responder al desafío que plantea el argumento diacrónico de Moretti es mostrar que solamente un sistema lógico puede superar el desafío que plantea su argumento sincrónico. Solo puede haber una lógica. El "Hay" de "Hay principios lógicos" debe ser el único "Hay" que hay.

Esto no significa olvidar que, para el naturalismo, "no hay manera de anticipar la forma en que se proyectarán las prácticas intersubjetivas (en particular, la práctica de elaborar creencias sobre el mundo)". Pero señalé dos párrafos atrás que el núcleo de la teoría lógica, en esta versión del naturalismo que estoy proponiendo, no se encuentra en la justificación de los principios lógicos sino en la demarcación de lógico y 
lo no lógico. Lo que hace naturalista a esta propuesta es su fidelidad a la idea de que la justificación de los principios lógicos se debe a la contribución que hacen a la derivación de resultados empíricos. Pero eso es compatible con que la frontera entre lo lógico y no lógico permanezca estable. La evolución de la "práctica de elaborar creencias sobre el mundo" podría encoger o agrandar el ámbito de lo que se reconoce como lógico, pero no podría mover la línea que divide lo lógico de lo no lógico. Una línea que solo se manifestará a quienes logren construir "una teoría lógico-semántica general, aplicable también al lenguaje de la teoría mis$m a$ ", lo que solo podrán hacer quienes hayan reconocido esa única lógica con la que se puede construir tal teoría.

No creo que esta breve y apurada descripción de la versión del proyecto naturalista que defiendo consiga convencer a alguien de su viabilidad técnica (no he dicho nada para justificar mi convicción de que es posible caracterizar un conjunto como $R_{L}$ que satisfaga todas las condiciones mencionadas) o de su interés filosófico. Solo espero que alcance para testimoniar cuánto le debe mi trabajo al estímulo generado por las ideas de Alberto Moretti.

\section{Bibliografía}

Moretti, A. (2006). Lógica y semántica. Revista de Filosofía. 31(2), 31-43. Moretti, A. (2016). La lógica y la trama de las cosas. Ideas y Valores, 65 (161), 5-22. 